\title{
新型查尔酮衍生物的合成及抗肿瘤活性评价
}

\author{
盛琦威赵婉秋曾明谢中袍夏雅平崔冬梅* \\ (浙江工业大学药学院 杭州 310014)
}

\begin{abstract}
摘要 主要通过 Claisen-Schmidt 缩合反应设计合成了一系列含有哌啶、吗啡啉、 $N$-甲基哌嗪取代的查尔酮衍生物，并 通过噻唑蓝(MTT) 法对 MCF-7(人乳腺癌细胞系)、A549(人肺癌细胞系)、HL-60(人白血病细胞系)、Hela(人宫颈癌细胞 系)和 Bewo(人线毛肿瘤细胞系)等五种癌细胞系进行了体外抗癌活性研究. 研究表明, 化合物 $\mathbf{4 a}, \mathbf{4 e}, \mathbf{4 f}, \mathbf{4 j}, \mathbf{4 m}, \mathbf{4 o}$ 对 MCF-7, A549, HL-60 三种癌细胞系抗癌活性最佳, $\mathrm{IC}_{50}$ 值均在 $10 \mu \mathrm{mol} / \mathrm{L}$ 以下.

关键词 查尔酮; 缩合; 抗癌; MTT 法
\end{abstract}

\section{Synthesis and Evaluation of Chalcone Derivatives as Novel Anticancer Agents}

\author{
Sheng, Qiwei Zhao, Wanqiu Zeng, Ming Xie, Zhongpao \\ Xia, Yaping Cui, Dongmei* \\ (College of Pharmaceutical Science, Zhejiang University of Technology, Hangzhou 310014)
}

\begin{abstract}
Three series of chalcones bearing a piperidino, morpholino, and 1-methylpiperazino moiety were synthesized in two steps with the key step being Claisen-Schmidt condensation and tested for the activity against five cell lines, MCF-7 (human breast adenocarcinoma cell line), A549 (human lung adenocarcinoma epithelial cell line), HL-60 (human leukemia cell line), Hela (human cervical cancer cell line), and Bewo (human chorionic tumor cell line) by thiazolyl blue tetrazolium bromide (MTT) assay. Some chalcones exhibited good anticancer activity, and among them $\mathbf{4 a}, \mathbf{4 e}, \mathbf{4 f}, \mathbf{4 j}, \mathbf{4 m}$, and $\mathbf{4 o}$ displayed the best anticancer activity for MCF-7 breast cancer cells, A549 lung cancer cells, and HL-60 leukemia cancer cells with IC 50 values below $10 \mu \mathrm{mol} / \mathrm{L}$, respectively.

Keywords chalcone; condensation; anticancer agents; MTT assay
\end{abstract}

\section{Introduction}

Chalcones, analogs of 1,3-diaryl prop-2-ene-1-one containing two aromatic rings bound with vinyl ketone fragment form a wide class of compounds, represent an interesting group of natural compounds that are abundant in fruits, vegetables, spices, tea, and soy based foodstuff and possess multifarious pharmacological potentials, such as anti-fungal, ${ }^{[1]}$ anti-hypertensive, ${ }^{[2]}$ hypnotic, ${ }^{[3]}$ antispasmodic, ${ }^{[4]}$ immunosuppressant, ${ }^{[5]}$ anti-platelet, ${ }^{[6]}$ antidiabetic, ${ }^{[7]}$ anti-tubercular, ${ }^{[8]}$ anti-neoplastic, ${ }^{[9]}$ antiangiogenic, ${ }^{[10]}$ anti-oxidant, ${ }^{[11]}$ hypolipidemic, ${ }^{[12]}$ anti-filarial, ${ }^{[13]}$ anti-retroviral, ${ }^{[14]}$ anti-malarial, ${ }^{[15]}$ antibacterial, ${ }^{[16]}$ anti-ulcer, ${ }^{[17]}$ anti-arrhythmic, ${ }^{[18]}$ anti-invasive, ${ }^{[19]}$ anti-histaminic, ${ }^{[20]}$ etc. The potential anticancer activities of chalcone derivatives has been explored. Recently, the anticancer activity of chalcones have been observed on inhib- iting various molecular targets, like ABCG2/P-gp/BCRP, $5 \alpha$-reductase, aromatase, 17- $\beta$-hydroxysteroid dehydrogenase, HDAC/Situin-1, proteasome, VEGF, VEGFR-2 kinase, MMP-2/9, JAK/STAT signaling pathways, CDC$25 \mathrm{~B}$, tubulin, cathepsin-K, topoisomerase-II, Wnt, NF- $\kappa \mathrm{B}$, B-Raf and mTOR. ${ }^{[9]}$ Heterocyclic nitrogen-containing substrates are common constituents of natural products, agrochemicals, and pharmaceuticals. Recently, the synthesis of hybrid compounds of chalcone and heterocycles, such as chalcone substituted piperazine, and their anticancer activity have been reported, but few chalcone substituted other heterocycles, for example piperidine, morpholine, have been documented in the literature. ${ }^{[21]}$ Based on the above observations, we have synthesized chalcone derivatives containing heterocyclic nitrogen. The synthesized compounds act as potential anticancer agents.

\footnotetext{
* Corresponding author. E-mail: cuidongmei@zjut.edu.cn

Received August 29, 2018; revised October 23, 2018; published online November 25, 2018.
} 


\section{Results and discussion}

\subsection{Synthesis}

The synthetic route of the proposed compounds $\mathbf{4 a} \sim \mathbf{4 s}$ is shown in Scheme 1. First, the acetophenones derivatives 3a $\sim 3 \mathbf{c}$ were obtained by the reaction of 4-fluorophenylethanone with piperidine, morpholine, and 1-methylpiperazine with stirring at reflux according to literature procedures. In the present investigation, substituted chalcones were prepared by the Claisen-Schmidt condensation of substituted 1-phenylethanone and substituted benzaldehyde with $\mathrm{NaOH}$ (aq.) as base in high yield by a known literature method. All chalcones were prepared from the corresponding reactants in 38\% $87 \%$ yields. Comparative data for novel derivatives with respective to structures, melting point and yield are provided in Table 1. A range of benzaldehydes, with electronrich or electron-poor substituents on the aryl rings at different positions, were well-tolerated for the condensation reaction, giving the corresponding products. The heterocyclic aldehydes were also found to be suitable reaction partners for oxygen- and nitrogencontaining aromatics, and the corresponding products were obtained.

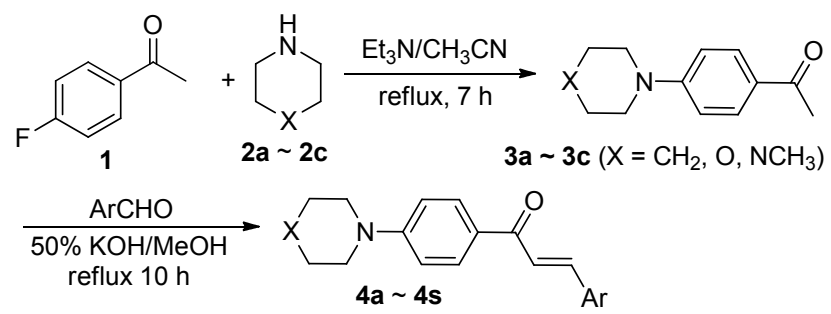

Scheme 1 Synthesis of chalcone derivatives $\mathbf{4 a} \sim \mathbf{4 s}$

Table 1 Physical data of compounds $4 \mathbf{a} \sim \mathbf{4 s}$

\begin{tabular}{|c|c|c|c|c|}
\hline \multicolumn{2}{|c|}{ Compd. X } & \multirow{2}{*}{$\frac{\mathrm{Ar}}{3,4-\left(\mathrm{OCH}_{2} \mathrm{O}\right) \mathrm{C}_{6} \mathrm{H}_{3}}$} & \multicolumn{2}{|c|}{ Yield $/ \%$ m.p. $/{ }^{\circ} \mathrm{C}$} \\
\hline $4 \mathbf{a}$ & $\mathrm{CH}_{2}$ & & 76 & $170 \sim 173$ \\
\hline $4 b$ & $\mathrm{CH}_{2}$ & $3-\mathrm{CH}_{3} \mathrm{O}-4-\mathrm{HOCH}_{2} \mathrm{CH}_{2} \mathrm{OC}_{6} \mathrm{H}_{3}$ & 65 & $167 \sim 170$ \\
\hline $4 c$ & $\mathrm{CH}_{2}$ & $3-\mathrm{CH}_{3} \mathrm{OC}_{6} \mathrm{H}_{4}$ & 44 & $160 \sim 163$ \\
\hline $4 d$ & $\mathrm{CH}_{2}$ & 4- $\left(\mathrm{CH}_{3}\right)_{2} \mathrm{NC}_{6} \mathrm{H}_{4}$ & 58 & $214 \sim 218$ \\
\hline $4 e$ & $\mathrm{CH}_{2}$ & 2-Furyl & 69 & $179 \sim 180$ \\
\hline $4 f$ & $\mathrm{CH}_{2}$ & 2-Pyridyl & 72 & $167 \sim 170$ \\
\hline $4 g$ & $\mathrm{O}$ & $3,4-\left(\mathrm{OCH}_{2} \mathrm{O}\right) \mathrm{C}_{6} \mathrm{H}_{3}$ & 76 & $167 \sim 171$ \\
\hline $4 h$ & $\mathrm{O}$ & $3-\mathrm{CH}_{3} \mathrm{O}-4-\mathrm{HOCH}_{2} \mathrm{CH}_{2} \mathrm{OC}_{6} \mathrm{H}_{3}$ & 41 & $165 \sim 168$ \\
\hline $4 i$ & $\mathrm{O}$ & $3-\mathrm{CH}_{3} \mathrm{OC}_{6} \mathrm{H}_{4}$ & 87 & $130 \sim 133$ \\
\hline $4 j$ & $\mathrm{O}$ & 4- $\left(\mathrm{CH}_{3}\right)_{2} \mathrm{NC}_{6} \mathrm{H}_{4}$ & 77 & $226 \sim 226$ \\
\hline $4 k$ & $\mathrm{O}$ & $4-\mathrm{BrC}_{6} \mathrm{H}_{4}$ & 79 & $213 \sim 216$ \\
\hline 41 & $\mathrm{O}$ & $2,4-\mathrm{Cl}_{2} \mathrm{C}_{6} \mathrm{H}_{3}$ & 40 & $147 \sim 148$ \\
\hline $4 m$ & $\mathrm{O}$ & 2-Furyl & 38 & $195 \sim 197$ \\
\hline $4 n$ & $\mathrm{O}$ & 2-Pyridyl & 52 & $150 \sim 153$ \\
\hline 40 & $\mathrm{CH}_{3} \mathrm{~N}$ & $3,4-\left(\mathrm{OCH}_{2} \mathrm{O}\right) \mathrm{C}_{6} \mathrm{H}_{3}$ & 52 & $155 \sim 158$ \\
\hline $4 p$ & $\mathrm{CH}_{3} \mathrm{~N}$ & $3-\mathrm{CH}_{3} \mathrm{O}-4-\mathrm{HOCH}_{2} \mathrm{CH}_{2} \mathrm{OC}_{6} \mathrm{H}_{3}$ & 54 & $185 \sim 188$ \\
\hline $4 q$ & $\mathrm{CH}_{3} \mathrm{~N}$ & $3-\mathrm{CH}_{3} \mathrm{OC}_{6} \mathrm{H}_{4}$ & 46 & $147 \sim 149$ \\
\hline $4 \mathbf{r}$ & $\mathrm{CH}_{3} \mathrm{~N}$ & 4- $\left(\mathrm{CH}_{3}\right)_{2} \mathrm{NC}_{6} \mathrm{H}_{4}$ & 57 & $198 \sim 201$ \\
\hline $4 s$ & $\mathrm{CH}_{3} \mathrm{~N}$ & 2-Furyl & 39 & $179 \sim 182$ \\
\hline
\end{tabular}

Reaction conditions: 3 (1.0 mmol), aldehyde (1.0 mmol), 50\% KOH (aq.) (1.0 $\mathrm{mL}), \mathrm{MeOH}(3 \mathrm{~mL})$, isolated yields.

\subsection{Spectra}

The structures of the obtained compounds $\mathbf{4 a} \sim \mathbf{4 s}$ were confirmed by ${ }^{1} \mathrm{H} \mathrm{NMR},{ }^{13} \mathrm{C}$ NMR and HRMS spectral data. In the ${ }^{1} \mathrm{H}$ NMR spectra of the compounds, the $\mathrm{CH}$ protons of the olefin resonated as a doublet at $\delta 7.58 \sim 8.17$ and $7.41 \sim 8.09$, respectively. The large coupling constant of the olefinic protons confirms the trans configuration. In general, IR spectra of these compounds exhibited the presence of absorption bands for $\mathrm{C}=\mathrm{O}$ between 1639 $1649 \mathrm{~cm}^{-1}$.

\subsection{Biological studies}

All the synthesized chalcone derivatives bearing a heterocyclic nitrogen moiety $\mathbf{4 a} \sim \mathbf{4 s}$ were evaluated for their anticancer activity in vitro against five cancer cell lines, MCF-7 (human breast adenocarcinoma cell line), A549 (human lung adenocarcinoma epithelial cell line), HL-60 (human leukemia cell line), Hela (human cervical cancer cell line), and Bewo (Human chorionic tumor cell line), using the 3-(4,5-dimethylthiazol-2-yl)-2,5-diphenyl tetrazolium bromide (MTT) assay method and compared with the well-known anticancer drug cisplatin. The anticancer activity results are shown in Table 2 . The results showed that the structures of compounds had an obvious influence on anticancer activities. Among all compounds, there were three series of substituents of core benzene ring, including piperidino, morpholino, and 1-methylpiperazino. In generally, derivatives containing piperidino were more potent than compounds with other substituted groups. For example, compound $\mathbf{4 a}$ displayed the best anticancer activity against five cancer cell lines [for $\mathrm{MCF}-7 \quad\left(\mathrm{IC}_{50}=8.70\right.$ $\mu \mathrm{mol} / \mathrm{L})$, A549 $\left(\mathrm{IC}_{50}=9.72 \mu \mathrm{mol} / \mathrm{L}\right), \mathrm{HL}-60\left(\mathrm{IC}_{50}=7.15\right.$ $\mu \mathrm{mol} / \mathrm{L})$, Hela $\left(\mathrm{IC}_{50}=12.88 \mu \mathrm{mol} / \mathrm{L}\right)$, and Bewo $\left(\mathrm{IC}_{50}=\right.$ $18.81 \mu \mathrm{mol} / \mathrm{L})]$. Among all synthesized derivatives, compound $\mathbf{4 j}$ showed the highest anticancer activity against MCF-7 with an $\mathrm{IC}_{50}$ value of $4.63 \mu \mathrm{mol} / \mathrm{L}$, and better than cisplatin against MCF-7 cells. Compound $\mathbf{4 j}$ also displayed the best anticancer activity for HL-60 cells $\left(\mathrm{IC}_{50}=6.00\right.$ $\mu \mathrm{mol} / \mathrm{L})$, compounds $\mathbf{4 e}, \mathbf{4 f}, \mathbf{4 m}$, and $4 \mathbf{0}$ also showed selected anticancer activity against HL-60 cell lines. According to the result, it is found that most chalcone derivatives were non-sensitive to Hela cells. Compound 4a showed the most anticancer activity $\left(\mathrm{IC}_{50}=12.88 \mu \mathrm{mol} / \mathrm{L}\right)$, and compounds $\mathbf{4 c}, \mathbf{4 d}, \mathbf{4 h}, \mathbf{4 j}$, and $\mathbf{4 n}$ had weak anticancer activity for Hela $\left(\mathrm{IC}_{50}<50 \mu \mathrm{mol} / \mathrm{L}\right)$. All compounds, except for $\mathbf{4 a}$, showed anticancer activity against Bewo cells at an $\mathrm{IC}_{50}$ level of $>50 \mu \mathrm{mol} / \mathrm{L}$.

The compounds $\mathbf{4 a} \sim \mathbf{4 e}$ containing piperidine group have significant potent anticancer activity $\left(\mathrm{IC}_{50}=8.70 \sim\right.$ $10.78 \mu \mathrm{mol} / \mathrm{L})$ against MCF-7 as compared to the other compounds $\mathbf{4 g} \sim \mathbf{4 i}, \mathbf{4 m}$ and $\mathbf{4 o} \sim \mathbf{4 s}$ containing morpholine or methylpiperazine groups as shown in Table 2. These data indicated that the activity of compounds $4 \mathbf{a} \sim \mathbf{4 e}$ was considerably attributed to the presence of piperidine group. The compound 4a with substituted $\mathrm{C}-3$ phenyl ring $[3,4-$ $\left.\left(\mathrm{OCH}_{2} \mathrm{O}\right)\right]$ showed to have good activity $\left(\mathrm{IC}_{50}=8.70\right.$ $\mu \mathrm{mol} / \mathrm{L})$. The change of $3,4-\left(\mathrm{OCH}_{2} \mathrm{O}\right)$ group to $3-\mathrm{CH}_{3} \mathrm{O}$ 
4- $\mathrm{HOCH}_{2} \mathrm{CH}_{2} \mathrm{O}, 3-\mathrm{CH}_{3} \mathrm{O}$, and $4-\left(\mathrm{CH}_{3}\right)_{2} \mathrm{~N}$ did not show good activity. The activity of compound $4 \mathrm{e}$ which $\mathrm{C}-3$ phenyl ring was changed to furanyl ring was similar to that of compound 4a. In the case of HL-60, investigation of structure-activity relationship (SAR) revealed that compounds 44, 4e, 4f, 4j, 4m and $40\left(\mathrm{IC}_{50}<10.0 \mu \mathrm{mol} / \mathrm{L}\right)$ have a significant potent activity against HL-60 as compared to the standard drug cisplatin $\left(\mathrm{IC}_{50}=2.90 \mu \mathrm{mol} / \mathrm{L}\right)$ as shown in Table 2. This potency due to the presence of $3,4-\left(\mathrm{OCH}_{2} \mathrm{O}\right)$ and $4-\left(\mathrm{CH}_{3}\right)_{2} \mathrm{~N}$ group at $\mathrm{C}-3$ phenyl ring in compounds $\mathbf{4 a}, \mathbf{4 j}$ and $\mathbf{4 o}\left(\mathrm{IC}_{50}=7.15,6.00\right.$ and 8.15 $\mu \mathrm{mol} / \mathrm{L})$, or furyl and pyridyl ring at $\mathrm{C}-3$ in compounds $4 \mathbf{e}$ and $4 \mathbf{f}\left(\mathrm{IC}_{50}=8.77\right.$ and $\left.9.62 \mu \mathrm{mol} / \mathrm{L}\right)$. In the case of $\mathrm{A} 549$, Hela, and Bewo, compound $\mathbf{4 a}$ also retained the activity $\left(\mathrm{IC}_{50}=9.72,12.88\right.$ and $\left.18.81 \mu \mathrm{mol} / \mathrm{L}\right)$, and the other compounds showed lower or no anticancer activity in three cell lines. It indicated the importance of the type of piperidine group and 3,4-( $\left.\mathrm{OCH}_{2} \mathrm{O}\right)$-substituted group.

Table 2 In vitro anticancer activity of the title compounds $\mathbf{4 a} \sim$ 4s against MCF-7, A549, HL-60, Hela, and Bewo cancer cell lines

\begin{tabular}{cccccc}
\hline \multirow{2}{*}{ Compd. } & \multicolumn{5}{c}{$\mathrm{IC}_{50}{ }^{a} /\left(\mu \mathrm{mol} \cdot \mathrm{L}^{-1}\right)$} \\
\cline { 2 - 6 } & MCF-7 & A549 & HL-60 & Hela & Bewo \\
\hline $\mathbf{4 a}$ & 8.70 & 9.72 & 7.15 & 12.88 & 18.81 \\
$\mathbf{4 b}$ & 14.99 & $>50$ & 22.51 & $>50$ & $>50$ \\
$\mathbf{4 c}$ & 21.50 & $>50$ & 12.62 & 42.92 & $>50$ \\
$\mathbf{4 d}$ & 17.59 & 44.99 & 49.35 & 45.37 & $>50$ \\
$\mathbf{4 e}$ & 10.78 & $>50$ & 8.77 & $>50$ & $>50$ \\
$\mathbf{4 f}$ & 26.73 & $>50$ & 9.62 & $>50$ & $>50$ \\
$\mathbf{4 g}$ & $>50$ & $>50$ & $>50$ & $>50$ & $>50$ \\
$\mathbf{4 h}$ & 19.39 & 43.63 & 26.63 & 32.43 & $>50$ \\
$\mathbf{4 i}$ & $>50$ & $>50$ & 14.98 & $>50$ & $>50$ \\
$\mathbf{4 j}$ & 4.63 & 35.62 & 6.00 & 39.24 & $>50$ \\
$\mathbf{4 k}$ & $>50$ & $>50$ & $>50$ & $>50$ & $>50$ \\
$\mathbf{4 l}$ & $>50$ & $>50$ & $>50$ & $>50$ & $>50$ \\
$\mathbf{4 m}$ & 15.69 & $>50$ & 9.11 & $>50$ & $>50$ \\
$\mathbf{4 n}$ & 16.58 & 44.19 & 11.37 & 25.29 & $>50$ \\
$\mathbf{4 0}$ & 39.46 & $>50$ & 8.15 & $>50$ & $>50$ \\
$\mathbf{4 p}$ & $>50$ & $>50$ & $>50$ & $>50$ & $>50$ \\
$\mathbf{4 q}$ & 23.52 & 45.49 & $>50$ & $>50$ & $>50$ \\
$\mathbf{4 r}$ & $>50$ & $>50$ & 24.86 & $>50$ & $>50$ \\
$\mathbf{4 s}$ & 24.43 & $>50$ & 13.67 & $>50$ & $>50$ \\
Cisplatin & 8.75 & 2.65 & 2.90 & 0.59 & 2.24 \\
\hline
\end{tabular}

${ }^{a}$ Anticancer activity was assayed by exposure for $48 \mathrm{~h}$ to the tested substances and expressed as the concentration required to inhibit tumour cell proliferation by $50 \%\left(\mathrm{IC}_{50}\right)$.

\section{Conclusions}

In summary, a series of chalcone derivatives have been synthesized and the effects of all the compounds on anticancer activity of five cancer cell lines (MCF-7, A549, HL-60, Hela, and Bewo) were investigated. Compounds 4a, $\mathbf{4 e}, \mathbf{4 f}, \mathbf{4 j}, \mathbf{4 m}$, and $4 \mathrm{o}$ displayed the best anticancer activity for MCF-7 breast cancer cells, A549, lung cancer cells, and HL-60 leukemia cancer cells, respectively $\left(\mathrm{IC}_{50}<10\right.$ $\mu \mathrm{mol} / \mathrm{L})$. Moreover, compound 4a had inhibitory effects on the growth of five cancer cell lines.

\section{Experimental}

\subsection{Experimental details}

Under otherwise noted, materials were obtained from Aladdin Co., Ltd. and used without further purification. Thin layer chromatography (TLC) was performed using silica gel 60 F254 and visualized using UV light. Column chromatography was performed with silica gel (mesh $300 \sim 400)$. NMR spectra were obtained on a Bruker Avance 500 spectrometer $\left({ }^{1} \mathrm{H}\right.$ NMR at $500 \mathrm{~Hz},{ }^{13} \mathrm{C}$ NMR at $125 \mathrm{~Hz}$ ) in $\mathrm{CDCl}_{3}$ or DMSO- $d_{6}$ using TMS as an internal standard. High-resolution mass spectra (HRMS) were measured on a high resolution mass spectrometer (GCT Premier). Infrared spectra (IR) were obtained on a 370 FT-IR spectrometer.

\subsection{4-Subtitued acetophenone 3a〜 3c}

These were synthesized as described in previous work. ${ }^{[22]}$

\subsection{General procedure for synthesis of $(E)$-chal- cones (4)}

To a mixture of acetophenone $(1.0 \mathrm{mmol})$ and aldehyde $(1.0 \mathrm{mmol})$ in methanol $(10 \mathrm{~mL})$ was added $50 \% \mathrm{KOH}$ (aq.) $(1 \mathrm{~mL})$. The resulting mixture was stirred for $10 \mathrm{~h}$ at reflux. The reaction mixture was cooled to room temperature. After evaporation of methanol and added water, the mixture was extracted with ethyl acetate. The organic phase was dried over anhydrous $\mathrm{Na}_{2} \mathrm{SO}_{4}$. The crude residue was obtained after evaporation of the solvent in vacuum, and the residue was purified by flash chromatography with petroleum and EtOAc as the eluent to give the pure product.

(E)-3-(1,3-Benzodioxol-5-yl)-1-(4-piperidinyl)phenyl-2propen-1-one (4a): 1-(4-(Piperidin-1-yl)phenyl)ethan-1one $(204.1 \mathrm{mg}, 1.0 \mathrm{mmol})$ and benzo[d][1,3]dioxole-5carbaldehyde $(149.9 \mathrm{mg}, 1.0 \mathrm{mmol})$ in methanol $(10 \mathrm{~mL})$ was reacted according to the general procedure for compounds 4 to give the product $4 \mathbf{a}(253.2 \mathrm{mg}, 76 \%$ yield $)$. $R_{\mathrm{f}}=0.45$ [ $V$ (petroleum ether $): V($ ethyl acetate $\left.)=3: 1\right]$. Yellow solid, m.p. $170 \sim 173{ }^{\circ} \mathrm{C} ;{ }^{1} \mathrm{H}$ NMR $(500 \mathrm{MHz}$, $\left.\mathrm{CDCl}_{3}\right) \delta: 7.98(\mathrm{~d}, J=9.0 \mathrm{~Hz}, 2 \mathrm{H}), 7.72(\mathrm{~d}, J=15.5 \mathrm{~Hz}$, $1 \mathrm{H}), 7.42(\mathrm{~d}, J=15.5 \mathrm{~Hz}, 1 \mathrm{H}), 7.18(\mathrm{~d}, J=1.3 \mathrm{~Hz}, 1 \mathrm{H}), 7.12$ (dd, $J=8.0,1.3 \mathrm{~Hz}, 1 \mathrm{H}), 6.91(\mathrm{~d}, J=9.0 \mathrm{~Hz}, 2 \mathrm{H}), 6.85$ (d, $J=8.0 \mathrm{~Hz}, 1 \mathrm{H}), 6.03(\mathrm{~s}, 2 \mathrm{H}), 3.39$ (t, $J=4.9 \mathrm{~Hz}, 2 \mathrm{H})$, $1.68 \sim 1.70(\mathrm{~m}, 6 \mathrm{H}) ;{ }^{13} \mathrm{C} \mathrm{NMR}\left(100 \mathrm{MHz}, \mathrm{CDCl}_{3}\right) \delta: 187.4$, $154.0,149.2,148.1,142.4,130.5,129.7,127.3,124.6$, $120.0,113.3,108.5,106.5,101.4,48.6,25.5,24.5$; IR (KBr) $v: 2935,1651,1600,1499,1360,1196,1124,1034 \mathrm{~cm}^{-1}$. HRMS (ESI) calcd for $\mathrm{C}_{21} \mathrm{H}_{22} \mathrm{NO}_{3}[\mathrm{M}+\mathrm{H}]^{+}:$226.1600, found 226.1603 .

(E)-3-[(3-Methoxyphenyl-4-(2-hydroxyethoxy)]-1-(4piperidinyl)phenyl-2-propen-1-one $(\mathbf{4 b}): R_{\mathrm{f}}=0.2[\mathrm{~V}$ (petroleum ether $): V($ ethyl acetate $)=1: 1]$. Yellow solid, m.p. $167 \sim 170{ }^{\circ} \mathrm{C} ;{ }^{1} \mathrm{H}$ NMR $\left(500 \mathrm{MHz}, \mathrm{CDCl}_{3}\right) \delta: 7.99(\mathrm{~d}, J=$ $8.9 \mathrm{~Hz}, 2 \mathrm{H}), 7.73$ (d, $J=15.5 \mathrm{~Hz}, 1 \mathrm{H}), 7.45$ (d, $J=15.5 \mathrm{~Hz}$, 
$1 \mathrm{H}), 7.21(\mathrm{dd}, J=8.3,1.4 \mathrm{~Hz}, 1 \mathrm{H}), 7.16(\mathrm{~d}, J=1.4 \mathrm{~Hz}, 1 \mathrm{H})$, $6.93(\mathrm{~d}, J=8.3 \mathrm{~Hz}, 1 \mathrm{H}), 6.90(\mathrm{~d}, J=8.9 \mathrm{~Hz}, 2 \mathrm{H}), 4.18(\mathrm{t}$, $J=4.4 \mathrm{~Hz}, 2 \mathrm{H}), 4.00 \sim 3.99(\mathrm{~m}, 2 \mathrm{H}), 3.93(\mathrm{~s}, 3 \mathrm{H}), 3.39(\mathrm{t}$, $J=5.4 \mathrm{~Hz}, 4 \mathrm{H}), 2.79(\mathrm{~s}, 1 \mathrm{H}), 1.69 \sim 1.67(\mathrm{~m}, 6 \mathrm{H}) ;{ }^{13} \mathrm{C} \mathrm{NMR}$ $\left(125 \mathrm{MHz}, \mathrm{CDCl}_{3}\right) \delta: 187.9,150.1,149.9,142.8,130.8$, $129.4,122.5,120.6,114.2,113.5,110.9,71.1,61.3,56.0$, 48.7, 25.4, 24.4; IR (KBr) v: 3537, 2939, 1643, 1609, 1511, $1268,1207,1033 \mathrm{~cm}^{-1}$. HRMS (ESI) calcd for $\mathrm{C}_{23} \mathrm{H}_{28} \mathrm{NO}_{4}$ $[\mathrm{M}+\mathrm{H}]^{+}:$382.2018; found 382.2014.

(E)-3-(3-Methoxyphenyl)-1-(4-piperidinyl)phenyl-2propen-1-one $(\mathbf{4 c )}): R_{\mathrm{f}}=0.25$ [ $V$ (petroleum ether) $: V($ ethyl acetate $)=10: 1)$. Yellow solid, m.p. $160 \sim 163{ }^{\circ} \mathrm{C} ;{ }^{1} \mathrm{H}$ NMR $\left(500 \mathrm{MHz}, \mathrm{CDCl}_{3}\right) \delta: 8.00(\mathrm{~d}, J=8.9 \mathrm{~Hz}, 2 \mathrm{H}), 7.76$ $(\mathrm{d}, J=15.6 \mathrm{~Hz}, 1 \mathrm{H}), 7.56(\mathrm{~d}, J=15.6 \mathrm{~Hz}, 1 \mathrm{H}), 7.34(\mathrm{t}, J=$ $7.8 \mathrm{~Hz}, 1 \mathrm{H}), 7.25$ (d, $J=7.8 \mathrm{~Hz}, 1 \mathrm{H}), 7.17$ (d, $J=2.1 \mathrm{~Hz}$, $1 \mathrm{H}), 6.96 \sim 6.92(\mathrm{~m}, 3 \mathrm{H}), 3.87(\mathrm{~s}, 3 \mathrm{H}), 3.41(\mathrm{t}, J=4.9 \mathrm{~Hz}$, $4 \mathrm{H}), 1.66 \sim 1.72(\mathrm{~m}, 6 \mathrm{H}) ;{ }^{13} \mathrm{C}$ NMR $\left(125 \mathrm{MHz}, \mathrm{CDCl}_{3}\right) \delta$ : $187.9,159.9,142.8,136.9,130.9,129.9,122.6,120.9$, $115.8,113.5,113.4,55.4,48.7,25.4,24.4$; IR (KBr) v: $3420,2945,2849,1649,1592,1384,1251,1127,1040 \mathrm{~cm}^{-}$ ${ }^{1}$. HRMS (ESI) calcd for $\mathrm{C}_{21} \mathrm{H}_{24} \mathrm{NO}_{2}[\mathrm{M}+\mathrm{H}]^{+}: 322.1807$, found 322.1809 .

(E)-3-[(4-Dimethylamino)phenyl]-1-(4-piperidinyl)phenyl-2-propen-1-one (4d): $R_{\mathrm{f}}=0.23 \quad[V($ petroleum ether $): V($ ethyl acetate $)=1: 1]$. Yellow solid, m.p. 214 $218{ }^{\circ} \mathrm{C} ;{ }^{1} \mathrm{H}$ NMR $\left(500 \mathrm{MHz}, \mathrm{CDCl}_{3}\right) \delta: 8.00(\mathrm{~d}, J=8.8 \mathrm{~Hz}$, $2 \mathrm{H}), 7.79(\mathrm{~d}, J=15.4 \mathrm{~Hz}, 1 \mathrm{H}), 7.56(\mathrm{~d}, J=8.8 \mathrm{~Hz}, 2 \mathrm{H}), 7.41$ $(\mathrm{d}, J=15.4 \mathrm{~Hz}, 1 \mathrm{H}), 6.91(\mathrm{~d}, J=8.8 \mathrm{~Hz}, 2 \mathrm{H}), 6.71(\mathrm{~d}, J=$ $8.8 \mathrm{~Hz}, 2 \mathrm{H}), 3.38(\mathrm{t}, J=5.1 \mathrm{~Hz}, 4 \mathrm{H}), 3.04(\mathrm{~s}, 6 \mathrm{H}), 1.74 \sim$ $1.62(\mathrm{~m}, 6 \mathrm{H}) ;{ }^{13} \mathrm{C}$ NMR $\left(125 \mathrm{MHz}, \mathrm{CDCl}_{3}\right) \delta: 188.2,151.7$, $143.8,130.4,130.0,123.3,117.1,113.6,111.9,48.8,40.2$, 25.4, 24.3; IR (KBr) v: 3408, 2933, 2854, 1732, 1641, 1599, $1228,1029 \mathrm{~cm}^{-1}$. HRMS (ESI) calcd for $\mathrm{C}_{22} \mathrm{H}_{27} \mathrm{~N}_{2} \mathrm{O}[\mathrm{M}+$ $\mathrm{H}]^{+}:$335.2123, found 335.2118.

(E)-3-(3-Furan-2-yl)-1-(4-piperidinyl)phenyl-2-propen1-one $(4 \mathbf{e}): R_{\mathrm{f}}=0.32[V($ petroleum ether $): V($ ethyl acetate $)=5: 1]$. Yellow solid, m.p. $179 \sim 180{ }^{\circ} \mathrm{C} ;{ }^{1} \mathrm{H}$ NMR $(500$ $\left.\mathrm{MHz}, \mathrm{CDCl}_{3}\right) \delta: 8.01(\mathrm{~d}, J=8.9 \mathrm{~Hz}, 2 \mathrm{H}), 7.58(\mathrm{~d}, J=15.5$ $\mathrm{Hz}, 1 \mathrm{H}), 7.52 \sim 7.48(\mathrm{~m}, 2 \mathrm{H}), 6.98 \sim 6.84(\mathrm{~m}, 2 \mathrm{H}), 6.68(\mathrm{~d}$, $J=3.4 \mathrm{~Hz}, 1 \mathrm{H}), 6.52 \sim 6.50(\mathrm{~m}, 1 \mathrm{H}), 3.41(\mathrm{t}, J=4.1 \mathrm{~Hz}$, $4 \mathrm{H}), 1.69 \sim 1.62(\mathrm{~m}, 6 \mathrm{H}) ;{ }^{13} \mathrm{C}$ NMR $\left(125 \mathrm{MHz}, \mathrm{CDCl}_{3}\right) \delta$ : $187.3,152.1,144.4,130.7,129.1,119.6,115.1,113.5$, $112.5,48.7,25.4,24.3$; IR (KBr) v: 3429, 2940, 2922, 2854, $1647,1603,1387,1234,1194,1123,1020 \mathrm{~cm}^{-1}$. HRMS (ESI) calcd for $\mathrm{C}_{18} \mathrm{H}_{20} \mathrm{NO}_{2}[\mathrm{M}+\mathrm{H}]^{+}$: 282.1494; found 282.1490 .

(E)-3-(Pyridin-2-yl)-1-(4-piperidinyl)phenyl-2-propen1-one (4f): $R_{\mathrm{f}}=0.25$ [ $V$ (petroleum ether) : $V($ ethyl acetate $)=3: 1]$. Yellow solid, m.p. $167 \sim 170{ }^{\circ} \mathrm{C} ;{ }^{1} \mathrm{H}$ NMR $(500$ $\left.\mathrm{MHz}, \mathrm{CDCl}_{3}\right) \delta: 8.70(\mathrm{~d}, J=4.0 \mathrm{~Hz}, 1 \mathrm{H}), 8.17(\mathrm{~d}, J=15.2$ $\mathrm{Hz}, 1 \mathrm{H}), 8.07$ (d, $J=9.0 \mathrm{~Hz}, 2 \mathrm{H}), 7.76(\mathrm{~d}, J=15.2 \mathrm{~Hz}, 1 \mathrm{H})$, $7.76 \sim 7.72(\mathrm{~m}, 1 \mathrm{H}), 7.47(\mathrm{~d}, J=7.8 \mathrm{~Hz}, 1 \mathrm{H}), 7.29 \sim 7.27$ $(\mathrm{m}, 1 \mathrm{H}), 6.90(\mathrm{~d}, J=9.0 \mathrm{~Hz}, 2 \mathrm{H}), 3.41(\mathrm{t}, J=4.8 \mathrm{~Hz}, 4 \mathrm{H})$, $1.70 \sim 1.68(\mathrm{~m}, 6 \mathrm{H}) ;{ }^{13} \mathrm{C}$ NMR $\left(125 \mathrm{MHz}, \mathrm{CDCl}_{3}\right) \delta: 187.6$, $154.4,153.7,150.0,140.8,136.8,131.1,126.9,125.8$, 125.1, 123.9, 113.2, 48.4, 25.3, 24.3; IR (KBr) v: 3421, 2934, 1647, 1588, 1392, 1192, 1126, $994 \mathrm{~cm}^{-1}$. HRMS
(ESI) calcd for $\mathrm{C}_{19} \mathrm{H}_{21} \mathrm{~N}_{2} \mathrm{O}[\mathrm{M}+\mathrm{H}]^{+}$: 293.1654; found 293.1655 .

(E)-3-(1,3-Benzodioxol-5-yl)-1-[4-(4-morpholinyl)phenyl]-2-propen-1-one (4g) (CAS: 305859-40-9): $R_{\mathrm{f}}=0.19$ $[V$ (petroleum ether) : $V$ (ethyl acetate $)=3: 1]$. Yellow solid, m.p. 167 171 ${ }^{\circ} \mathrm{C} ;{ }^{1} \mathrm{H}$ NMR $\left(500 \mathrm{MHz}, \mathrm{CDCl}_{3}\right) \delta$ : $8.01(\mathrm{~d}, J=9.0 \mathrm{~Hz}, 2 \mathrm{H}), 7.74$ (d, $J=15.5 \mathrm{~Hz}, 1 \mathrm{H}), 7.42$ (d, $J=15.5 \mathrm{~Hz}, 1 \mathrm{H}), 7.19(\mathrm{~d}, J=1.5 \mathrm{~Hz}, 1 \mathrm{H}), 7.13(\mathrm{dd}, J=8.0$, $1.5 \mathrm{~Hz}, 1 \mathrm{H}), 6.93(\mathrm{~d}, J=9.0 \mathrm{~Hz}, 2 \mathrm{H}), 6.86(\mathrm{~d}, J=8.0 \mathrm{~Hz}$, $1 \mathrm{H}), 6.04(\mathrm{~s}, 2 \mathrm{H}), 3.89$ (t, $J=4.9 \mathrm{~Hz}, 4 \mathrm{H}), 3.35$ (t, $J=4.9$ $\mathrm{Hz}, 4 \mathrm{H})$.

(E)-3-[(3-Methoxyphenyl-4-(2-hydroxyethoxy)]-1-[4(4-morpholinyl)phenyl]-2-propen-1-one $(\mathbf{4 h}): \quad R_{\mathrm{f}}=0.13$ $[V$ (petroleum ether $): V($ ethyl acetate $)=1: 1]$. Yellow solid, m.p. $165 \sim 168{ }^{\circ} \mathrm{C} ;{ }^{1} \mathrm{H}$ NMR $\left(500 \mathrm{MHz}, \mathrm{CDCl}_{3}\right) \delta$ : $8.02(\mathrm{~d}, J=8.8 \mathrm{~Hz}, 2 \mathrm{H}), 7.75(\mathrm{~d}, J=15.6 \mathrm{~Hz}, 1 \mathrm{H}), 7.44$ (d, $J=15.6 \mathrm{~Hz}, 1 \mathrm{H}), 7.23(\mathrm{dd}, J=8.2,1.6 \mathrm{~Hz}, 1 \mathrm{H}), 7.17(\mathrm{~d}$, $J=1.6 \mathrm{~Hz}, 1 \mathrm{H}), 6.96 \sim 6.92(\mathrm{~m}, 3 \mathrm{H}), 4.19(\mathrm{t}, J=4.4 \mathrm{~Hz}$, $2 \mathrm{H}), 4.01 \sim 3.99(\mathrm{~m}, 2 \mathrm{H}), 3.95(\mathrm{~s}, 3 \mathrm{H}), 3.88(\mathrm{t}, J=4.8 \mathrm{~Hz}$, $4 \mathrm{H}), 3.35(\mathrm{t}, J=4.8 \mathrm{~Hz}, 4 \mathrm{H}), 2.54(\mathrm{t}, J=6.1 \mathrm{~Hz}, 1 \mathrm{H}) ;{ }^{13} \mathrm{C}$ NMR $\left(125 \mathrm{MHz}, \mathrm{CDCl}_{3}\right) \delta$ : 188.2, 154.0, 150.2, 149.9, 143.3, 130.6, 129.2, 122.5, 120.4, 114.1, 113.6, 110.9, 71.1, 66.6, 61.2, 56.0, 47.7; IR (KBr) v: 3539, 2966, 2858, 1641, 1607, 1508, 1266, $1032 \mathrm{~cm}^{-1}$. HRMS (ESI) calcd for $\mathrm{C}_{22} \mathrm{H}_{26} \mathrm{NO}_{5}[\mathrm{M}+\mathrm{H}]^{+}: 384.1811$; found 384.1808.

(E)-3-(3-Methoxyphenyl)-1-[4-(4-morpholinyl)phenyl]2-propen-1-one (4i) (CAS: 850351-83-6): $\quad R_{\mathrm{f}}=0.2$ $[V$ (petroleum ethe) : $V($ ethyl acetate $)=3: 1]$. Yellow solid, m.p. $130 \sim 133{ }^{\circ} \mathrm{C} ;{ }^{1} \mathrm{H}$ NMR $\left(500 \mathrm{MHz} \mathrm{CDCl}_{3}\right) \delta$ : $8.03(\mathrm{~d}, J=8.9 \mathrm{~Hz}, 2 \mathrm{H}), 7.77$ (d, $J=15.6 \mathrm{~Hz}, 1 \mathrm{H}), 7.55$ (d, $J=15.6 \mathrm{~Hz}, 1 \mathrm{H}), 7.34(\mathrm{t}, J=7.8 \mathrm{~Hz}, 1 \mathrm{H}), 7.26(\mathrm{~d}, J=7.8$ $\mathrm{Hz}, 1 \mathrm{H}), 7.17(\mathrm{~s}, 1 \mathrm{H}), 6.97 \sim 6.94(\mathrm{~m}, 1 \mathrm{H}), 6.93$ (d, $J=8.9$ $\mathrm{Hz}, 2 \mathrm{H}), 3.89 \sim 3.88(\mathrm{~m}, 4 \mathrm{H}), 3.87(\mathrm{~s}, 3 \mathrm{H}), 3.35(\mathrm{t}, J=5.0$ $\mathrm{Hz}, 4 \mathrm{H})$.

(E)-3-[(4-Dimethylamino)phenyl]-1-[4-(4-morpholinyl)phenyl]-2-propen-1-one (4j) (CAS: 1004038-07-6): $R_{\mathrm{f}}=$ $0.35[V$ (petroleum ether) $: V($ ethyl acetate $)=2: 1]$; yellow solid, m.p. 226 228 ${ }^{\circ} \mathrm{C}$; ${ }^{1} \mathrm{H}$ NMR (500 MHz, $\left.\mathrm{CDCl}_{3}\right) \delta$ 8.02 (d, $J=8.9 \mathrm{~Hz}, 2 \mathrm{H}), 7.80$ (d, $J=15.4 \mathrm{~Hz}, 1 \mathrm{H}), 7.56$ (d, $J=8.8 \mathrm{~Hz}, 2 \mathrm{H}), 7.39$ (d, $J=15.4 \mathrm{~Hz}, 1 \mathrm{H}), 6.93$ (d, $J=8.9$ $\mathrm{Hz}, 2 \mathrm{H}), 6.71(\mathrm{~d}, J=8.8 \mathrm{~Hz}, 2 \mathrm{H}), 3.88(\mathrm{t}, J=4.9 \mathrm{~Hz}, 4 \mathrm{H})$, $3.35(\mathrm{t}, J=4.9 \mathrm{~Hz}, 4 \mathrm{H}), 3.05(\mathrm{~s}, 6 \mathrm{H})$.

(E)-3-(4-Bromophenyl)-1-[4-(4-morpholinyl)phenyl]-2propen-1-one $(\mathbf{4 k})^{[23]}: \quad R_{\mathrm{f}}=0.26[V$ (petroleum ether) : $V($ ethyl acetate $)=3: 1]$. Yellow solid, m.p. $213 \sim 216{ }^{\circ} \mathrm{C}$; ${ }^{1} \mathrm{H}$ NMR $\left(500 \mathrm{MHz}, \mathrm{CDCl}_{3}\right) \delta: 8.02(\mathrm{~d}, J=9.0 \mathrm{~Hz}, 2 \mathrm{H})$, $7.73(\mathrm{~d}, J=15.6 \mathrm{~Hz}, 1 \mathrm{H}), 7.57 \sim 7.50(\mathrm{~m}, 5 \mathrm{H}), 6.93(\mathrm{~d}, J=$ $9.0 \mathrm{~Hz}, 2 \mathrm{H}), 3.88(\mathrm{t}, J=4.9 \mathrm{~Hz}, 4 \mathrm{H}), 3.55(\mathrm{t}, J=4.9 \mathrm{~Hz}$, $4 \mathrm{H})$.

(E)-3-(2,4-Dichlorophenyl)-1-[4-(4-morpholinyl)phenyl]-2-propen-1-one (4I) (CAS: 796989-79-2): $R_{\mathrm{f}}=0.33$ $[V$ (petroleum ether) $: V($ ethyl acetate $)=3: 1]$. Yellow solid, m.p. 147 148 ${ }^{\circ} \mathrm{C} ;{ }^{1} \mathrm{H}$ NMR $\left(500 \mathrm{MHz}, \mathrm{CDCl}_{3}\right) \delta$ : $8.09(\mathrm{~d}, J=15.7 \mathrm{~Hz}, 1 \mathrm{H}), 8.01(\mathrm{~d}, J=8.9 \mathrm{~Hz}, 2 \mathrm{H}), 7.69$ (d, $J=8.5 \mathrm{~Hz}, 1 \mathrm{H}), 7.51(\mathrm{~d}, J=15.7 \mathrm{~Hz}, 1 \mathrm{H}), 7.48$ (d, $J=2.0$ $\mathrm{Hz}, 1 \mathrm{H}), 7.31$ (dd, $J=8.5,2.0 \mathrm{~Hz}, 1 \mathrm{H}), 6.93(\mathrm{~d}, J=8.9 \mathrm{~Hz}$, 2H), 3.89 (t, $J=5.0 \mathrm{~Hz}, 4 \mathrm{H}), 3.36$ (t, $J=5.0 \mathrm{~Hz}, 4 \mathrm{H})$. 
(E)-3-(3-Furan-2-yl)-1-[4-(4-morpholinyl)phenyl]-2propen-1-one (4m): $R_{\mathrm{f}}=0.3$ [ $V$ (petroleum ether) $: V($ ethyl acetate $)=3: 1]$. Yellow solid, m.p. $195 \sim 197{ }^{\circ} \mathrm{C} ;{ }^{1} \mathrm{H}$ NMR (500 MHz, $\left.\mathrm{CDCl}_{3}\right) \delta: 8.03(\mathrm{~d}, J=9.0 \mathrm{~Hz}, 2 \mathrm{H}), 7.59$ $(\mathrm{d}, J=15.3 \mathrm{~Hz}, 1 \mathrm{H}), 7.53(\mathrm{~d}, J=1.7 \mathrm{~Hz}, 1 \mathrm{H}), 7.50$ (d, $J=$ $15.3 \mathrm{~Hz}, 1 \mathrm{H}), 6.94$ (d, $J=9.0 \mathrm{~Hz}, 2 \mathrm{H}), 6.70$ (d, $J=3.4 \mathrm{~Hz}$, $1 \mathrm{H}), 6.52$ (dd, $J=3.4,1.7 \mathrm{~Hz}, 1 \mathrm{H}), 3.89$ (t, $J=4.9 \mathrm{~Hz}, 4 \mathrm{H})$, $3.35(\mathrm{t}, J=4.9 \mathrm{~Hz}, 4 \mathrm{H}) ;{ }^{13} \mathrm{C} \mathrm{NMR}\left(125 \mathrm{MHz}, \mathrm{CDCl}_{3}\right) \delta$ : $187.6,154.1,152.0,144.5,130.6,129.5,128.9,119.4$, 115.4, 113.5, 112.5, 66.6, 47.6; IR (KBr) v: 3457, 2974, 2850, 1647, 1604, 1384, 1231, 1193, 1130, $1025 \mathrm{~cm}^{-1}$. HRMS (ESI) calcd for $\mathrm{C}_{17} \mathrm{H}_{18} \mathrm{NO}_{3}[\mathrm{M}+\mathrm{H}]^{+}$: 284.1287; found 284.1287

(E)-3-(Pyridin-2-yl)-1-[4-(4-morpholinyl)phenyl]-2propen-1-one (4n): $R_{\mathrm{f}}=0.41$ [ $V$ (petroleum ether) $: V($ ethyl acetate $)=3: 1]$. Yellow solid, m.p. $150 \sim 153{ }^{\circ} \mathrm{C} ;{ }^{1} \mathrm{H}$ NMR (500 MHz, $\left.\mathrm{CDCl}_{3}\right) \delta: 8.69(\mathrm{~d}, J=3.9 \mathrm{~Hz}, 1 \mathrm{H}), 8.15$ (d, $J=15.2 \mathrm{~Hz}, 1 \mathrm{H}), 8.09$ (d, $J=9.0 \mathrm{~Hz}, 2 \mathrm{H}), 7.76$ (d, $J=$ $15.2 \mathrm{~Hz}, 1 \mathrm{H}), 7.75 \sim 7.72(\mathrm{~m}, 1 \mathrm{H}), 7.47(\mathrm{~d}, J=7.7 \mathrm{~Hz}, 1 \mathrm{H})$, $7.30 \sim 7.28(\mathrm{~m}, 1 \mathrm{H}), 6.91(\mathrm{~d}, J=9.0 \mathrm{~Hz}, 2 \mathrm{H}), 3.87(\mathrm{t}, J=4.9$ $\mathrm{Hz}, 4 \mathrm{H}), 3.35(\mathrm{t}, J=4.9 \mathrm{~Hz}, 4 \mathrm{H}) ;{ }^{13} \mathrm{C} \mathrm{NMR}(125 \mathrm{MHz}$, $\left.\mathrm{CDCl}_{3}\right) \delta: 188.0,154.3,153.6,150.1,141.3,136.9,130.9$, 128.6, 125.7, 125.3, 124.1, 113.3, 66.6, 47.4; IR (KBr) v: 3463, 2960, 1656, 1591, 1382, 1193, 1117, $1049 \mathrm{~cm}^{-1}$. HRMS (ESI) calcd for $\mathrm{C}_{18} \mathrm{H}_{19} \mathrm{~N}_{2} \mathrm{O}_{2}[\mathrm{M}+\mathrm{H}]^{+}$: 295.1447 ; found 295.1448 .

(E)-3-(1,3-Benzodioxol-5-yl)-1-[4-(4-methylpiperazin1-yl)phenyl]-2-propen-1-one (4o): $R_{\mathrm{f}}=0.45$ (ethyl acetate). Yellow solid, m.p. $155 \sim 158{ }^{\circ} \mathrm{C} ;{ }^{1} \mathrm{H}$ NMR $(500 \mathrm{MHz}$, $\left.\mathrm{CDCl}_{3}\right) \delta: 8.00(\mathrm{~d}, J=8.9 \mathrm{~Hz}, 2 \mathrm{H}), 7.73(\mathrm{~d}, J=15.5 \mathrm{~Hz}$, $1 \mathrm{H}), 7.41(\mathrm{~d}, J=15.5 \mathrm{~Hz}, 1 \mathrm{H}), 7.18(\mathrm{~d}, J=1.6 \mathrm{~Hz}, 1 \mathrm{H}), 7.13$ $(\mathrm{dd}, J=8.0,1.6 \mathrm{~Hz}, 1 \mathrm{H}), 6.93(\mathrm{~d}, J=8.9 \mathrm{~Hz}, 2 \mathrm{H}), 6.85$ (d, $J=8.0 \mathrm{~Hz}, 1 \mathrm{H}), 6.03$ (s, 2H), 3.42 (t, $J=4.9 \mathrm{~Hz}, 4 \mathrm{H}), 2.60$ $(\mathrm{t}, J=4.9 \mathrm{~Hz}, 4 \mathrm{H}), 2.39(\mathrm{~s}, 3 \mathrm{H}) ;{ }^{13} \mathrm{C} \mathrm{NMR}(100 \mathrm{MHz}$, $\left.\mathrm{CDCl}_{3}\right) \delta 187.6,153.7,149.3,148.1,142.8,130.4,129.7$, $128.3,124.7,119.9,113.5,108.5,106.5,101.4,54.7,47.3$, 46.1; IR (KBr) v: 3418, 2904, 1647, 1609, 1384, 1250, $1198,1037,810 \mathrm{~cm}^{-1}$. HRMS (ESI) calcd for $\mathrm{C}_{21} \mathrm{H}_{23} \mathrm{~N}_{2} \mathrm{O}_{3}$ $[\mathrm{M}+\mathrm{H}]^{+}: 351.1709$; found 351.1711 .

(E)-3-[(3-Methoxyphenyl-4-(2-hydroxyethoxy)]-1-[4(4-methylpiperazin-1-yl)phenyl]-2-propen-1-one (4p): $R_{\mathrm{f}}=0.18$ (ethyl acetate). Yellow solid, m.p. $185 \sim 188{ }^{\circ} \mathrm{C}$; ${ }^{1} \mathrm{H}$ NMR $\left(500 \mathrm{MHz}, \mathrm{CDCl}_{3}\right) \delta: 8.01(\mathrm{~d}, J=8.9 \mathrm{~Hz}, 2 \mathrm{H})$, $7.75(\mathrm{~d}, J=15.6 \mathrm{~Hz}, 1 \mathrm{H}), 7.44(\mathrm{~d}, J=15.6 \mathrm{~Hz}, 1 \mathrm{H}), 7.23$ $(\mathrm{dd}, J=8.3,1.7 \mathrm{~Hz}, 1 \mathrm{H}), 7.17(\mathrm{~d}, J=1.7 \mathrm{~Hz}, 1 \mathrm{H}), 6.95 \sim$ $6.92(\mathrm{~m}, 3 \mathrm{H}), 4.19(\mathrm{t}, J=4.5 \mathrm{~Hz}, 2 \mathrm{H}), 4.00(\mathrm{t}, J=4.5 \mathrm{~Hz}$, $2 \mathrm{H}), 3.94(\mathrm{~s}, 3 \mathrm{H}), 3.42(\mathrm{t}, J=4.9 \mathrm{~Hz}, 4 \mathrm{H}), 2.59(\mathrm{t}, J=4.9$ $\mathrm{Hz}, 4 \mathrm{H}), 2.38(\mathrm{~s}, 3 \mathrm{H}) ;{ }^{13} \mathrm{C} \mathrm{NMR}\left(125 \mathrm{MHz}, \mathrm{CDCl}_{3}\right) \delta 188.2$, $154.1,150.2,149.9,143.2,130.7,129.3,128.6,122.5$, $120.5,114.1,113.7,110.9,71.1,61.3,56.0,54.8,47.3$, 46.1; IR (KBr) v: 3414, 2939, 2844, 1644, 1599, 1511, $1269,1141,1032 \mathrm{~cm}^{-1}$. HRMS (ESI) calcd for $\mathrm{C}_{23} \mathrm{H}_{29} \mathrm{~N}_{2} \mathrm{O}_{4}$ $[\mathrm{M}+\mathrm{H}]^{+}:$397.2127; found 397.2123.

(E)-3-(3-Methoxyphenyl)-1-[4-(4-methylpiperazin-1-yl) phenyl]-2-propen-1-one (4q): $R_{\mathrm{f}}=0.21$ (ethyl acetate). Yellow solid, m.p. $147 \sim 149{ }^{\circ} \mathrm{C} ;{ }^{1} \mathrm{H}$ NMR $(400 \mathrm{MHz}$,
$\left.\mathrm{CDCl}_{3}\right) \delta: 7.97(\mathrm{~d}, J=8.9 \mathrm{~Hz}, 2 \mathrm{H}), 7.73(\mathrm{~d}, J=15.6 \mathrm{~Hz}$, $1 \mathrm{H}), 7.52(\mathrm{~d}, J=15.6 \mathrm{~Hz}, 1 \mathrm{H}), 7.31(\mathrm{t}, J=7.7 \mathrm{~Hz}, 1 \mathrm{H}), 7.22$ $(\mathrm{d}, J=7.7 \mathrm{~Hz}, 1 \mathrm{H}), 7.13(\mathrm{t}, J=1.9 \mathrm{~Hz}, 1 \mathrm{H}), 6.93 \sim 6.92(\mathrm{~m}$, $1 \mathrm{H}), 6.90$ (d, $J=8.9 \mathrm{~Hz}, 2 \mathrm{H}), 3.85$ (s, 3H), 3.39 (t, $J=4.9$ $\mathrm{Hz}, 4 \mathrm{H}), 2.56(\mathrm{t}, J=4.9 \mathrm{~Hz}, 4 \mathrm{H}), 2.36(\mathrm{~s}, 3 \mathrm{H}) ;{ }^{13} \mathrm{C} \mathrm{NMR}$ $\left(125 \mathrm{MHz} \mathrm{CDCl}_{3}\right) \delta: 187.7,159.7,153.8,142.8,136.5$, $130.5,129.7,128.1,122.2,120.8,115.6,113.4,113.2,55.4$, 54.7, 47.3, 46.2; IR (KBr) v: 3473, 2936, 1660, 1603, 1382, $1250,1203,1053 \mathrm{~cm}^{-1}$. HRMS (ESI) calcd for $\mathrm{C}_{21} \mathrm{H}_{25} \mathrm{~N}_{2} \mathrm{O}_{2}$ $[\mathrm{M}+\mathrm{H}]^{+}:$337.1916; found 337.1919.

(E)-3-[(4-Dimethylamino)phenyl]-1-[4-(4-methylpiperazin-1-yl)phenyl]-2-propen-1-one $(4 \mathrm{r}): R_{\mathrm{f}}=0.19$ (ethyl acetate). Yellow solid, m.p. $198 \sim 201{ }^{\circ} \mathrm{C} ;{ }^{1} \mathrm{H}$ NMR $(500$ $\left.\mathrm{MHz}, \mathrm{CDCl}_{3}\right) \delta: 8.01(\mathrm{~d}, J=8.9 \mathrm{~Hz}, 2 \mathrm{H}), 7.79$ (d, $J=15.4$ $\mathrm{Hz}, 1 \mathrm{H}), 7.56$ (d, $J=8.8 \mathrm{~Hz}, 2 \mathrm{H}), 7.39$ (d, $J=15.4 \mathrm{~Hz}, 1 \mathrm{H})$, $6.93(\mathrm{~d}, J=8.9 \mathrm{~Hz}, 2 \mathrm{H}), 6.71(\mathrm{~d}, J=8.8 \mathrm{~Hz}, 2 \mathrm{H}), 3.40(\mathrm{t}$, $J=5.0 \mathrm{~Hz}, 4 \mathrm{H}), 3.04(\mathrm{~s}, 6 \mathrm{H}), 2.58(\mathrm{t}, J=5.0 \mathrm{~Hz}, 4 \mathrm{H}), 2.37$ $(\mathrm{s}, 3 \mathrm{H}) ;{ }^{13} \mathrm{C} \mathrm{NMR}\left(100 \mathrm{MHz}, \mathrm{CDCl}_{3}\right) \delta: 188.0,153.5,151.5$, $143.9,130.2$, 129.9, 129.0, 122.9, 116.8, 113.5, 111.7, 54.8, 47.4, 46.2, 40.2; IR (KBr) v: 3455, 2936, 2795, 1636, 1598, $1518,1231,1180,1033 \mathrm{~cm}^{-1}$. HRMS (ESI) calcd for $\mathrm{C}_{22} \mathrm{H}_{28} \mathrm{~N}_{3} \mathrm{O}[\mathrm{M}+\mathrm{H}]^{+}: 350.2232$; found 350.2231.

(E)-3-(3-Furan-2-yl)-1-[4-(4-methylpiperazin-1-yl)phenyl]-2-propen-1-one (4s): $R_{\mathrm{f}}=0.15$ (ethyl acetate). Yellow solid, m.p. $179 \sim 182{ }^{\circ} \mathrm{C}$; ${ }^{1} \mathrm{H}$ NMR (400 MHz, $\left.\mathrm{CDCl}_{3}\right) \delta: 7.98(\mathrm{~d}, J=8.9 \mathrm{~Hz}, 2 \mathrm{H}), 7.55(\mathrm{~d}, J=15.3 \mathrm{~Hz}$, $1 \mathrm{H}), 7.49 \sim 7.44(\mathrm{~m}, 2 \mathrm{H}), 6.89(\mathrm{~d}, J=8.9 \mathrm{~Hz}, 2 \mathrm{H}), 6.65(\mathrm{~d}$, $J=3.3 \mathrm{~Hz}, 1 \mathrm{H}), 6.48(\mathrm{dd}, J=3.3,1.7 \mathrm{~Hz}, 1 \mathrm{H}), 3.39$ (t, $J=$ $4.9 \mathrm{~Hz}, 4 \mathrm{H}), 2.56(\mathrm{t}, J=4.9 \mathrm{~Hz}, 4 \mathrm{H}), 2.36(\mathrm{~s}, 3 \mathrm{H}) ;{ }^{13} \mathrm{C} \mathrm{NMR}$ $\left(125 \mathrm{MHz} \mathrm{CDCl}_{3}\right) \delta: 187.4,154.0,152.1,144.4,130.6$, $129.3,128.3,119.5,115.2,113.5,112.5,54.7,47.2,46.1$; IR $(\mathrm{KBr}) v: 3416,2845,1647,1602,1383,1233,1187$, $1019,923 \mathrm{~cm}^{-1}$. HRMS (ESI) calcd for $\mathrm{C}_{18} \mathrm{H}_{21} \mathrm{~N}_{2} \mathrm{O}_{2}[\mathrm{M}+$ $\mathrm{H}]^{+}:$297.1603; found 297.1606.

\subsection{Cytotoxic activity}

For observing the anticancer activity of the target compounds in vitro, MTT assay was used. MCF-7, A549, HL-60, Hela, and Bewo cells were harvested in the logarithmic growth phase and seeded in 96-well plates, and cultured at $37{ }^{\circ} \mathrm{C}$ in humidified atmosphere containing $5 \% \mathrm{CO}_{2}$ in Dulbecco's Modified Eagle Medium (DMEM) with $10 \%$ FBS for $24 \mathrm{~h}$ before any treatments. Tested compounds were dissolved in dimethyl sulfoxide (DMSO) and diluted in the culture fluid to get various concentrations. The cells were treated with target compounds subsequently and incubated $24 \mathrm{~h}$. Then $20 \mu \mathrm{L}$ of MTT (5 $\mathrm{mg} / \mathrm{mL}$ ) was added in a $37{ }^{\circ} \mathrm{C}, 5 \% \mathrm{CO}_{2}$ incubator for $4 \mathrm{~h}$, the medium was removed immediately and MTT formazan was solubilized in $150 \mu \mathrm{L}$ of DMSO. Cell survival was assayed by enzyme-labeled instrument. Inhibitory effects were determined as $\mathrm{IC}_{50}$ value. ${ }^{[23]}$

Supporting Information The electronic supplementary information (ESI) available free of charge via the Internet at http://sioc-journal.cn/. 


\section{References}

[1] Lahtchev, K. L.; Batovska, D. I.; Parushev, S. P.; Ubiyvovk, V. M.; Sibirny, A. A. Eur. J. Med. Chem. 2008, 43, 2220.

[2] (a) Kantevari, S.; Addla, D.; Bagul, P. K.; Sridhar, B.; Banerjee, S. K. Bioorg. Med. Chem. 2011, 19, 4772.

(b) Yan, Y. K.; Xu, Q.; Gao, Y.; Liu, H.; Tang, X. R. Chin. J. Org. Chem. 2018, 38, 1763 (in Chinese).

(严映坤，徐侨，高扬，刘辉，唐孝荣，有机化学，2018，38，1763.)

[3] (a) Cho, S.; Kim, S.; Jin, Z.; Yang, H.; Han, D.; Baek, N. I.; Jo, J.; Cho, C. W.; Park, J. H.; Shimizu, M.; Jin, Y. H. Res. Commun. 2011, 413, 637.

(b) Zhang, E.; Wang, M. M.; Xu, S. M.; Wang, S.; Zhao, D.; Bai, P. Y.; Cui, D. Y.; Hua, Y. G.; Wang, Y. N.; Qin, S. S; Liu, H. M. Chin. J. Org. Chem. 2017, 37, 959 (in Chinese).

(张恩, 王铭铭, 徐帅民, 王上, 赵娣, 白鹏燕, 崔得运, 化永刚, 王亚娜, 秦上尚, 刘宏民, 有机化学, 2017, 37, 959.)

[4] Sato, Y.; He, J.; Nagai, H.; Tani, T.; Akao, T. Biol. Pharm. Bull. 2007, 30, 145

[5] Luo, Y.; Song, R.; Li, Y.; Zhang, S.; Liu, Z. J.; Fu, J.; Zhu, H. L. Bioorg. Med. Chem. Lett. 2012, 22, 3039.

[6] Zhao, L.; Jin, H.; Sun, L.; Piao, H.; Quan, Z. Bioorg. Med. Chem. Lett. 2005, 15, 5027.

[7] Mahapatra, D. K.; Asati, V.; Bharti, S. K. Eur. J. Med. Chem. 2015, $92,839$.

[8] Mascarello, A.; Chiaradia, L. D.; Vernal, J.; Villarino, A.; Guido, R. V.; Perizzolo, P.; Poirier, V.; Wong, D.; Martins, P. G.; Nunes, R. J.; Yunes, R. A.; Andricopulo, A. D.; Av-Gay, Y.; Terenzi, H. Bioorg. Med. Chem. 2010, 18, 3783.

[9] Mahapatra, D. K.; Bharti, S. K.; Asati, V. Eur. J. Med. Chem. 2015, $98,69$.

[10] Lee, Y. S.; Lim, S. S.; Shin, K. H.; Kim, Y. S.; Ohuchi, K.; Jung, S. H. Biol. Pharm. Bull. 2006, 29, 1028.

[11] Aoki, N.; Muko, M.; Ohta, E.; Ohta, S. J. Nat. Prod. 2008, 71, 1308 .
[12] Sashidhara, K. V.; Palnati, G. R.; Sonkar, R.; Avula, S. R.; Awasthi, C.; Bhatia, G. Eur. J. Med. Chem. 2013, 64, 422.

[13] Sashidhara, K. V.; Rao, K. B.; Kushwaha, V.; Modukuri, R. K.; Verma, R.; Murthy, P. K. Eur. J. Med. Chem. 2014, 81, 473.

[14] Rizvi, S. U. F.; Siddiqui, H. L.; Johns, M.; Detorio, M.; Schinazi, R. F. Med. Chem. Res. 2012, 21, 3741 .

[15] Tomar, V.; Bhattacharjee, G.; Kamaluddin; Rajakumar, S.; Srivastava, K.; Puri, S. K. Eur. J. Med. Chem. 2010, 45, 745.

[16] Abdullah, M. I.; Mahmood, A.; Madni, M.; Masood, S.; Kashif, M. Bioorg. Chem. 2014, 54, 31.

[17] Sashidhara, K. V.; Avula, S. R.; Mishra, V.; Palnati, G. R.; Singh, L. R.; Singh, N.; Chhonker, Y. S.; Swamy, P.; Bhatta, R. S.; Palit, G. Eur. J. Med. Chem. 2015, 89, 638.

[18] Yarishkin, O. V.; Ryu, H. W.; Park, J.; Yang, M. S.; Hong, S.; Park, K. H. Bioorg. Med. Chem. Lett. 2008, 18, 137.

[19] Wang, L.; Chen, G.; Lu, X.; Wang, S.; Hans, S.; Li, Y.; Ping, G.; Jiang, X.; Li, H.; Yang, J.; Wu, C. Eur. J. Med. Chem. 2015, 89, 88.

[20] Yamamoto, T.; Yoshimura, M.; Yamaguchi, F.; Kouchi, T.; Tsuji, R.; Saito, M.; Obata, A.; Kikuchi, M. Biosci. Biotechnol. Biochem. 2004, 68, 1706.

[21] Gao, H.; Zheng, X.; Qi, Y.; Wang, S.; Wan, C. P.; Rao, G. X. ; Mao, Z. W. Chin. J. Org. Chem. 2018, 38, 648 (in Chinese) (高慧, 郑喜, 祁燕, 王斯, 万春平, 饶高雄, 毛泽伟, 有机化学, 2018, 38, 648.)

[22] Thanusu, J.; Kanagarajan, V.; Gopalakrishnan, M. J. Enzyme Inhib. Med. Chem. 2010, 25, 347.

[23] Ramya, V.; Vembu, S.; Ariharasivakumar, G.; Gopalakrishnan, M. Pharma Chem. 2017, 9, 46

[24] Arasakumar, T.; Mathusalini, S.; Ata, A.; Shankar, R.; Gopalan, S.; Lakshmi, K.; Sakthivel, P.; Mohan, P. S. Mol. Diversity 2017, 21, 37.

[25] Zhang, X.; Shi, G.; He, L.; Tao, K.; Hou, T. Pesticides 2012, 51, 301 (in Chinese)

(张新刚，史冠芗，何利钦，陶科，侯太平，农药, 2012, 51,301.) 